\title{
Effects of Solder Voids on Microwave Transmission Performance in Passive Circuits
}

\author{
Qiu Yingxia ${ }^{1, a}$, Peng Xuelin ${ }^{2, b}$, Cheng Jingsheng ${ }^{2, c}$, and Min Zhixian ${ }^{1, \mathrm{~d}^{*}}$ \\ ${ }^{1}$ The 38th Research Institute China Electronics Technology Group Corporation Hefei, China, 230088 \\ ${ }^{2}$ Key Laboratory of Electronic Equipment Structure Design, Ministry of Education Xidian University \\ a helenqyx@163.com, b pengxuelinxd@163.com, 'dzixian_min@163.com
}

Keywords: soldering, void, grounding, signal transmission, HFSS.

\begin{abstract}
Grounding is an important technology of microwave circuits. Soldering process can realize the grounding of microwave chip. However, the solder voids in soldering can make the reliability of grounding worse, deteriorating heat conduction performance and conductive properties, resulting in an error in signal transmission and significantly reduce the transmission performance of microwave signal. As the device operating frequency goes higher, solder voids can lead to serious signal integrity problems, for this reason, influence of different positions, sizes and number of void in soldering on signal transmission performance of microwave were investigated. The result shows that the void close to the feeding port has the greatest influence on the signal transmission performance, the signal transmission performance worsens with the volume of void enlarging, in addition, below the transmission line, with the increasing in number of voids, the signal transmission performance is deteriorated.
\end{abstract}

\section{Introduction}

With the development of electronic technology, various high-speed RF circuit systems are widely used in many areas, such as communications, radar, surveillance and navigation. With the development of electronic devices trends towards light weight and miniaturization, arrangement of electronic components become more intensive, which puts forward a very high request on processing technology of microwave circuits. However, the structure and characteristic of RF circuits affect the signal transmission performance directly.

In a high-speed interconnection system, the signal transmits through the chip internal wiring, chip package, PCB board routing channels, the pad, through-hole, bolts, etc., the electrical characteristics of the signal itself makes it a fact that the signal integrity problems may exist in any transmission path. In assembly process of PCB, there are various connection techniques, such as welding, module assembly, bolt connections. In the past, the connection technique depends on the experience of technical personnel and lacks a theoretical guidance of its impact on signal transmission performance. With the deepening of the research on the electromechanical coupling of electronic equipment, the effect of PCB welding process on the electrical performance of microwave components has become one of the popular research topics.

Grounding is an important technology of microwave circuits, because the current needs to flow through the ground to form a loop. Soldering is mainly used in the circuit substrate between the bottom of the chip and the grounding shell to achieve the grounding and signal connection. Practical experience with projects has shown that it is very easy to produce solders voids in soldering process, which has become a very important uncertainty for long-term reliability of the chip. The presence of voids will cause deterioration of the reliability of the device ground and thermal conductivity performance, especially with the device operating frequency increases, soldering voids can cause serious signal integrity problems. Therefore, this paper studies the soldering process, the most typical in the connection techniques of microwave circuit, to analyze the effect of the characteristic of solder voids in soldering on microwave signal transmission performance. 


\section{Analytical methods and computational model}

Solder void is a hole in the solder layer, shown in Figure 3. During reflow soldering process, a large amount of gas in the solder material is wrapped without exclusion when the solder layer is formed, that is the direct reason for the cavitation of sold voids. These materials are usually solder solvent or other additives in solder, which can be volatilized out during the reflow process, not only that they can also be the gas which was generated when a chemical reaction occurs between metal pad on the surface of the circuit board, pin of the component, oxides on the surface of metal powder in solder and flux. The current study shows that a variety of welding materials in the welding process is the main reason for the formation of voids.

In order to grasp the effects of void size, location and number on signal transmission performance quantitatively. This paper takes the microwave printed circuit board as the research object (Fig. 1) to study the effects of different size, different position and different number of voids in the solder layer on signal transmission performance in high frequency.

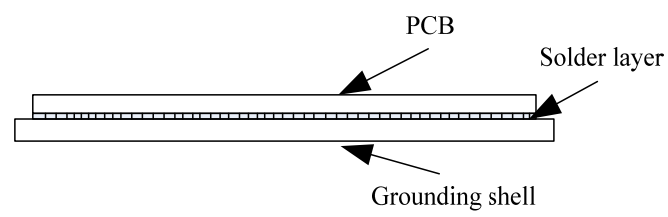

Fig. 1 Schematic diagram of a large area of soldering

Simulations and analysis with different circumstances of voids are carried out based on three-dimensional electromagnetic simulation software HFSS, and the electrical parameters of various model are obtained under different conditions. The method can be carried out according to the following steps: first, to establish the structural model of solder void in HFSS, and then define the boundary conditions of various parts, finally, calculate in the required frequency band, and obtain the electrical parameters of the signal transmission. When analyzing the other cases, we can simply change the position or size of the voids and so on, and adopt the same set of other structures and boundary conditions.

Establishing the model is the basis of studying the effects of solders voids on signal transmission performance, thus, to simplify the analysis, we created a microstrip transmission line model in HFSS, The model's geometry and material is shown in Table 1, the complete structural model is shown in figure 2 .

Table 1. Geometry and material of components

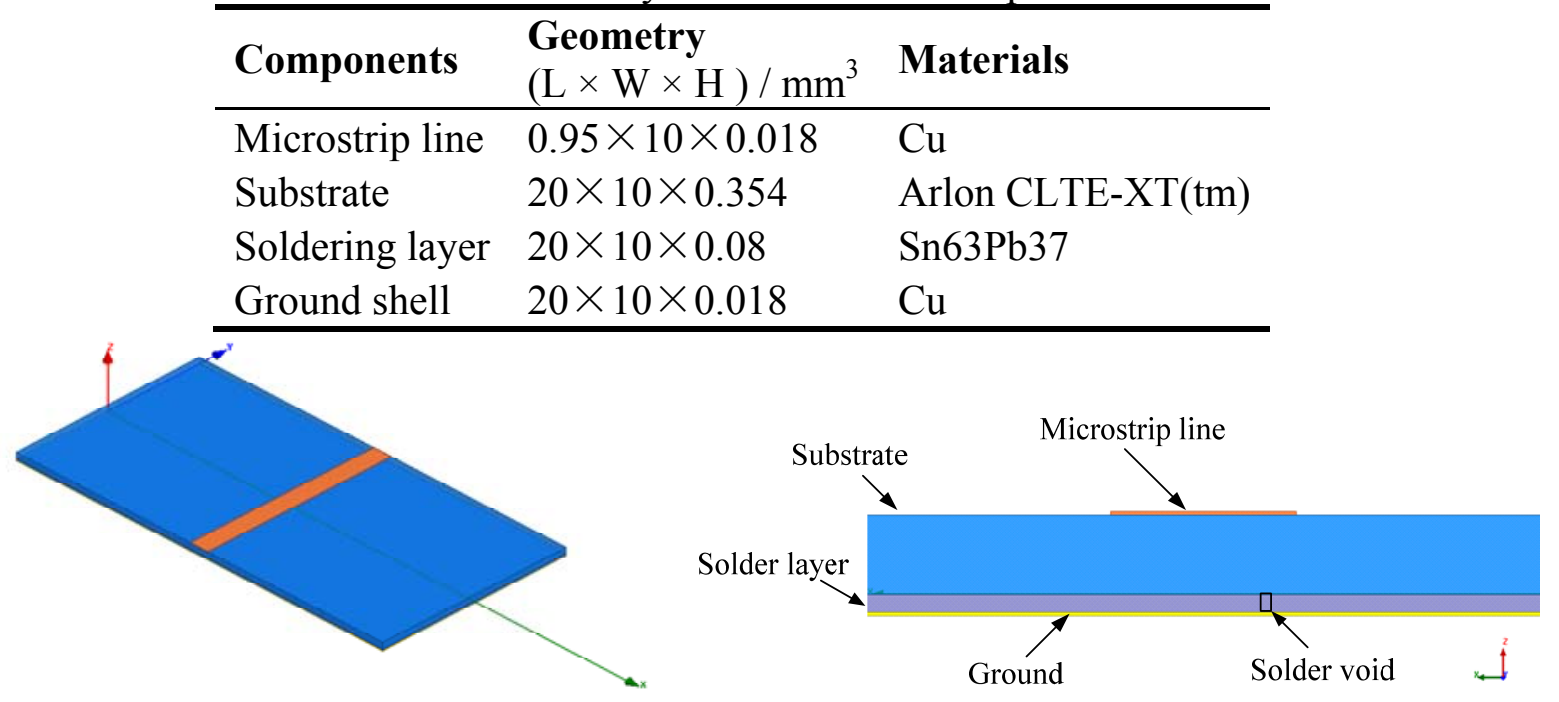

Fig. 2 Schematic diagram of microstrip line

Figure 3 is a schematic diagram of single void positions, the position (1) and (5) is a void at the port of the microstrip line, the position (2) and (3) is a void inside the solder layer, the position (4) is a void at the side position in the solder layer. 


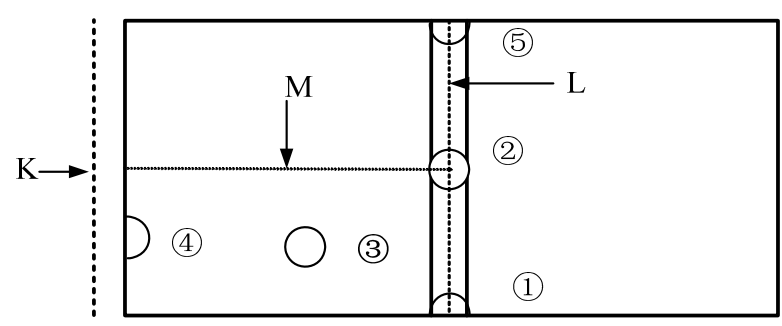

Fig. 3 Schematic diagram of void distribution

\section{Results and discussion}

Based on Figure 3, for the main parameters of position, number and diameter of void, we performed simulations and analysis on solder voids with conditions of different positions, different sizes and different numbers in HFSS, resulting in the main parameter of signal transmission performance - voltage standing wave ratio (VSWR).

The 3-D physical model of solder voids in HFSS, carried out simulations for the cases described above, and then calculated the electrical performance parameters of each microwave circuit model in a certain frequency range.

\section{Effects of different position of voids on signal transmission performance}

To study the effect of different positions of voids on the signal transmission performance, we selected five different positions (shown in Figure 3) in the solder layer, and built a hollow cylinder of which diameter is $1 \mathrm{~mm}$ and height is $0.08 \mathrm{~mm}$ respectively, to represent the solder void. Because of the similar influence of the position (1) and (5), we only chose four positions to analyze the effects of them, the results are shown in Figure 4.

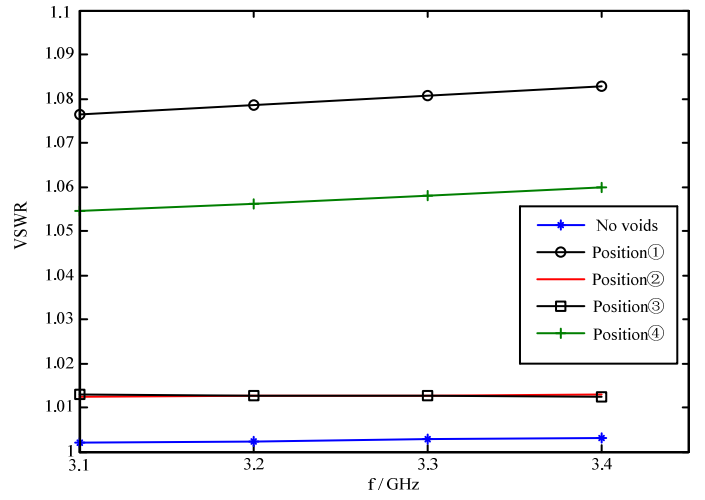

Fig. 4 VSWR of different locations

As can be seen from the Fig 4, the voids do have a certain impact on microwave signal transmission performance; the small to large order of the influence of voids positions is (3)(2)(4)(1). At the location of the feed port, the influence is max, but inside the solder layer, the influence is less.

To better illustrate the impact of different positions of solder voids on signal transmission performance, we analyzed the change in signal transmission performance, when the positions of solder voids change along different routes. In Figure 4, the dotted line L denotes a straight line connecting the front and rear feed port in the solder layer, the dotted line $\mathrm{M}$ denotes a horizontal line inside the solder layer, the dotted line $\mathrm{K}$ denotes a vertical line in the solder layer. At intervals of 1 $\mathrm{mm}$ along the line $\mathrm{L}, \mathrm{M}, \mathrm{K}$, we built a cylinder model of which diameter is $1 \mathrm{~mm}$, then performed the simulations respectively, and obtained the results as shown in Fig 5. In Fig 5, the abscissa means the position of the solder voids, for line $\mathrm{L}$ and $\mathrm{K}$, the origin of coordinate means the lower end of the solder layer, and the coordinate of 10 point means the upper end of the solder layer. For line $\mathrm{M}$, the origin of coordinate means the leftmost of line $\mathrm{M}$, that is the edge of the solder layer, and the coordinate of 10 point means the rightmost of line $\mathrm{M}$, that is the middle of the solder layer. The ordinate means VSWR of microstrip circuit with the frequency of $3.25 \mathrm{GHz}$. 


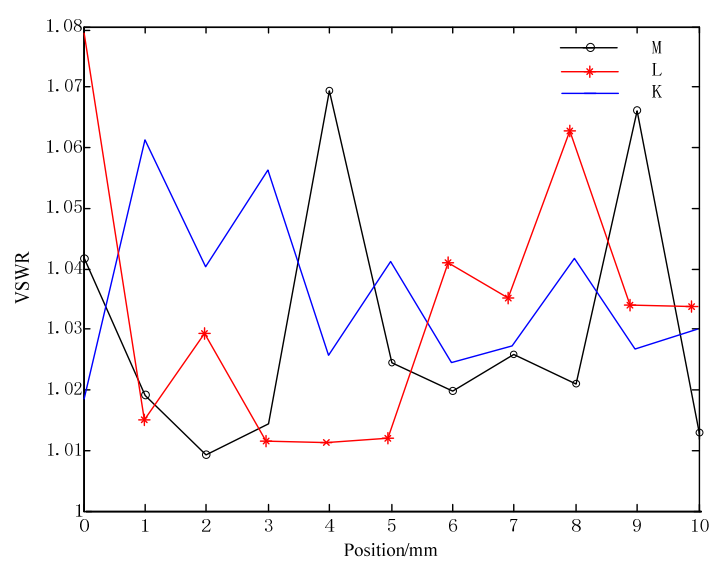

Fig. 5 VSWR along different routes

From Fig 5, it can be known: the coordinate value of 0 and 8 on the curve $\mathrm{L}$ means the greater values in VSWR, this shows that the voids at the location of feeding ports and output ports have the greater influence on signal transmission performance than others, and the influence of voids at the location of the feeding ports is the greatest. As can be seen from the curve $\mathrm{M}$, the influence of solder voids in the solder layer is hopping; the law of the impact is not obvious. The overall trend of curve $\mathrm{K}$ is decreased gradually, indicating that with the increase of the distance from solder voids to feeding port, the effect on signal transmission performance becomes smaller gradually.

\section{Effects of voids size on signal transmission performance}

In order to analyze the effects of solder void sizes on signal transmission performance, we choose 3 representative positions (1) (2) (4), and then built a cylinder model of which diameter is $1 \mathrm{~mm}-2 \mathrm{~mm}$, to perform the simulations respectively, and obtained the results as shown in Fig 6.

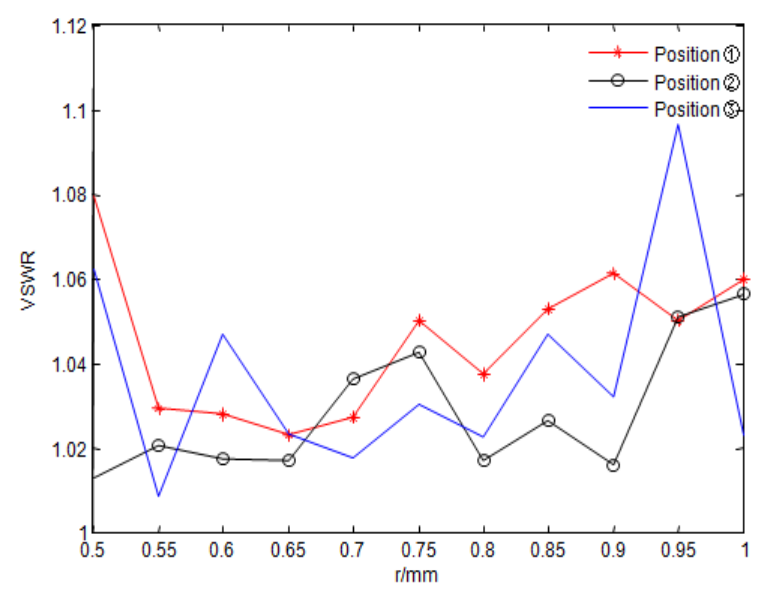

Fig. 6 VSWR under different size of solder voids

From Fig 6, it can be known: with the void diameter increases, the overall trend of VSWR is gradually increasing, but the effects of solder void sizes on signal transmission performance are not large as a whole, both are within the acceptable range in engineering.

\section{Effects of voids numbers on the signal transmission performance}

Here, we take the center position (2) as a reference, and increase the solder voids along two sides in the directions of vertical and horizontal (Figure 7) respectively, to study the effects of the change in the number of voids in both directions on signal transmission performance. The results obtained by simulations are shown in Fig 7. 


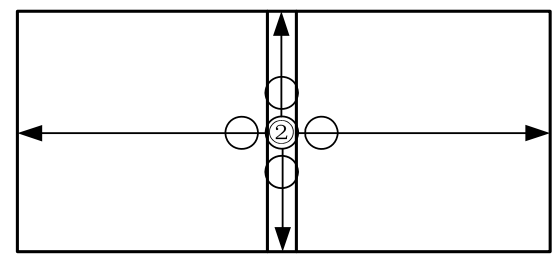

Fig. 7 Schematic diagram of the direction in voids increasing

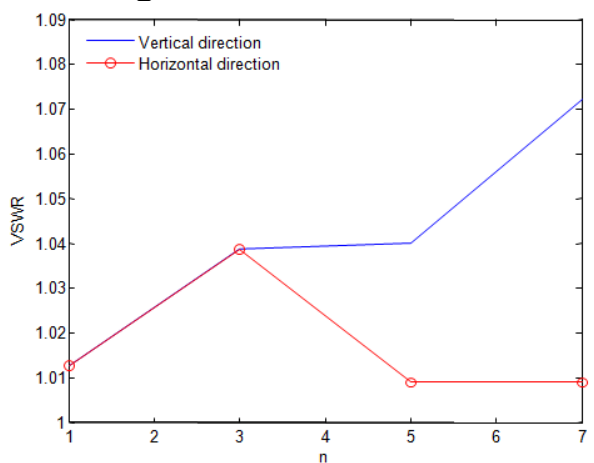

Fig. 8 VSWR under a different number of voids

As can be seen from Fig 8, the effect on signal transmission performance is different when the numbers of voids are different, in the vertical direction below the microstrip line, with the number of voids increases, the signal transmission performance is gradually deteriorated, however, as the number of voids increases in the horizontal direction, the VSWR did not change significantly. Therefore, with the number of voids beneath the microstrip line increases, the impact on signal transmission performance is obvious.

\section{Summary}

In this paper, we study the effects of solder voids on signal transmission performance and find that not only do the solder voids have effects on the quality and reliability of the solder, but they also have certain effects on signal transmission performance, especially in high-speed signal transmission, with the signal frequency increases, the effect is also more pronounced. The results can be drawn from this article: the voids at the feeding port have a greater influence on signal transmission performance than the internal voids; the greater the void volume is, the worsen the signal transmission performance is, however, in a certain range of the voids diameter, its impact can be ignored; in the transport direction, with the number of voids increases, the signal transmission performance will deteriorate.

Current research is limited to the simulation analysis, and there is still a certain distance from practical engineering applications. In the future, we will continue to carry out researches on quality evaluation in soldering from the following three aspects: (1) In practical engineering, the structures of solder voids vary, so the cylindrical voids are a little different from the actual voids. In the future work, we can refer to some actual solder voids, and summary the characteristics of voids model and improve them. (2) All of the solder form, package size, package materials have impacts on signal transmission performance, thus establishing realistic three-dimensional physical circuit model is the basis of research, the model in this paper is a microstrip line, and is different from the structure of the ground form of chips, so we will improve the existing circuit model and investigate the mechanism of impact in the solder connections more accurately. (3)As can be seen from the paper, the effects of solder voids in S-band on signal transmission performance is still relatively small, so in the future work, we will expand the frequency from S-band to X-band to study the effects in higher frequency

\section{References}


[1]. Kranenburg R A, Long S A. Microstrip transmission line excitation of dielectric resonator antennas[J]. Electronics Letters, 1988, 24(18): 1156-1157.

[2]. Lorrain P, Corson D. Electromagnetic fields and waves[J]. 1970

[3]. D. R. Shirley, G. Bendzsak, L. J. Turbini and G. Munie. Evaluating the effect of solder paste residues on RF signals between 5 and $10 \mathrm{GHz}$. SMTA Journal of SMT, 2004, 17(1): 25-32.

[4]. Zhang Ying, Janet M.Wang, Xiao Liang, Wu Hui-Zhong. A new stochastic modeling and analysis method for transmission lines in the presence of random process variations. Acta Electronica Sinica. 2005, 33(11): 1959-1964.

[5]. Mousskhani K, Ghorbani A. A modified broadband transmission line model for rectangular patch antennas. International Conference on Microwave and Millimeter Wave Technology, 21-24 April 2008

[6]. Said Al-Sarawi, Derek Abbott, Paul D Franzon. A review of 3D packaging technology. IEEE Transactions on Components Packaging and Manufacturing Technology Part B, 1998, 21(1): 2-14

[7]. M. Young, The Technical Writer's Handbook. Mill Valley, CA: University Science, 1989.

[8]. Electronic Publication: Digital Object Identifiers (DOIs):Article in a journal:

[9]. D. Kornack and P. Rakic, "Cell Proliferation without Neurogenesis in Adult Primate Neocortex," Science, vol. 294, Dec. 2001, pp. 2127-2130, doi:10.1126/science.1065467.Article in a conference proceedings:

[10]. H. Goto, Y. Hasegawa, and M. Tanaka, "Efficient Scheduling Focusing on the Duality of MPL Representatives," Proc. ASME Symp. Computational Intelligence in Scheduling (SCIS 07), ASME Press, Dec. 2007, pp. 57-64, doi:10.1109/SCIS.2007.357670. 